\title{
Tratamiento quirúrgico ganglionar regional en los pacientes con melanoma primario localizado y biopsia de ganglio centinela positivo: Conducta clínica en el Instituto Nacional de Cancerología, Colombia
}

\author{
Surgical regional lymph node treatment in patients with localized primary melanoma and \\ positive sentinel lymph node biopsy: Clinical management at the National Cancer Institute \\ of Colombia
}

Ivan Mariño-Lozano ${ }^{\mathrm{a}}$ (D) , Carlos Lehmann-Mosquera ${ }^{\mathrm{b}}$ (D), Mauricio Garcia-Morac (iD), Javier Angel-Aristizabal ${ }^{\mathrm{b}}$, Carlos Duarte-Torres $^{\mathrm{b}}$ (D) , Sergio Cervera-Bonilla ${ }^{\mathrm{b}}$ (D) , Patricia Lopez-Correa $^{\mathrm{d}}$ (D), Sandra Esperanza Diaz-Casas ${ }^{\mathrm{b}}$ (D)

\begin{abstract}
Sentinel lymph node status has been identified as the strongest prognostic factor for survival in patients with localized melanoma. There is controversy regarding surgical treatment versus observation in patients with a diagnosis of localized melanoma and tumor involvement in the sentinel lymph node. Traditionally, complete regional lymphadenectomy has been the standard management, however, this procedure has not shown benefits in melanoma-specific overall survival. Additionally, it is associated with greater morbidity and a delay in the initiation of systemic therapies with an impact on disease-free survival. A literature search was carried out in the two most important bibliographic databases as sources of clinical trials. Joint meetings were held between the functional units for breast and soft tissue tumors, clinical oncology, and pathology to establish recommendations for the therapeutic approach in relation to surgical regional lymph node treatment in patients with localized primary melanoma and positive sentinel lymph node biopsy. The decision to omit immediate regional lymphadenectomy in patients with localized cutaneous melanoma and lymph node tumor involvement in the sentinel lymph node biopsy must be individualized and made together with the patient, taking into account several aspects, including a complete pathology analysis of the surgical specimen, clinical and ultrasound follow-up of regional nodes, knowledge and awareness of the patient about their disease, early initiation of adjuvant systemic therapy, and the possibility of immediate therapeutic lymphadenectomy if regional relapse occurs.
\end{abstract}

Keywords: Melanoma, sentinel lymph node, lymph node excision.

\section{Resumen}

El estado del ganglio centinela se ha identificado como el factor pronóstico más fuerte de supervivencia en pacientes con diagnóstico de melanoma localizado. Existe controversia en el tratamiento quirúrgico versus la observación en los pacientes con diagnóstico de melanoma localizado y compromiso tumoral en el ganglio centinela. La conducta estándar, hasta hace un par de años, era la linfadenectomía regional completa; sin embargo, este procedimiento no ha demostrado beneficio en la supervivencia global específica por melanoma, pero si asociación con una mayor morbilidad y retraso en el inicio de terapias sistémicas que sí tienen impacto probado en superviviencia libre de enfermedad. Se realizó una búsqueda de la literatura en las dos bases de datos bibliográficas más importantes como fuentes de ensayos clínicos. Se realizaron reuniones conjuntas entre las unidades funcionales de mama y tejidos blandos, oncología clínica y patología, estableciendo conductas a seguir en el abordaje terapéutico en relación al tratamiento quirúrgico ganglionar regional en los pacientes con melanoma primario localizado y biopsia de ganglio centinela positivo. La decisión de omisión de linfadenectomía ganglionar regional inmediata, en los pacientes con melanoma cutáneo localizado y compromiso tumoral ganglionar en la biopsia de ganglio centinela, debe ser individualizada y en conjunto con el paciente, teniendo en cuenta el análisis completo de la pieza quirúrgica en patología, el inicio temprano de la terapia sistémica adyuvante, la garantía del seguimiento clínico y

\footnotetext{
a. Especialista en entrenamiento Cirugía de Mama y Tumores de Tejidos blandos, FUCS - INC, Bogotá, Colombia

b. Especialista en Cirugía de Mama y Tumores Tejidos Blandos, Unidad funcional de Mama y Tumores de Tejidos Blandos, Instituto Nacional de Cancerología Bogotá, Colombia

c. Especiálista en Cirugía Oncológica, Unidad funcional de Mama y Tejidos Blandos, Instituto Nacional de Cancerología, Bogotá, Colombia

d. Especialista en Patología Oncológica, Instituto Nacional de Cancerología, Bogotá, Colombia
}

Autor para correspondencia: Sandra E. Díaz Casas Correo electrónico: sdiaz@cancer.gov.co 
ecográfico de las cadenas ganglionares regionales, todo lo cual requiere conocimiento y concientizacion del paciente sobre su enfermedad, y sobre la posibilidad de realizar linfadenectomía terapéutica inmediata, de llegar a presentarse recaída regional.

Palabras clave: melanoma, ganglio linfático centinela, escisión del ganglio linfático

\section{Introducción}

Según datos de GLOBOCAN, para el año 2020 la incidencia y mortalidad de melanoma por 100.000 habitantes en el mundo fue 3.4 y 0.56 respectivamente (1). Las tasas de mortalidad por cáncer de piel atribuidas al melanoma maligno alcanzan $60 \%$ (1). La incidencia estimada del melanoma maligno en Colombia corresponde a 4,6 por 100.000 en mujeres y 4,4 por 100.000 en hombres (2).

El compromiso ganglionar en los pacientes con melanoma se ha considerado un factor de mal pronóstico desde sus reportes iniciales (3). Históricamente, la linfadenectomía regional electiva inmediata se consideró como parte importante en el tratamiento quirúrgico de esta enfermedad, incluso en la ausencia de compromiso ganglionar clínicamente evidente $(4,5)$. Lo anterior se realizaba basándose en la premisa de que la resección temprana de las cadenas ganglionares regionales permitiría eliminar la enfermedad antes de que esta se diseminará más allá de los ganglios linfáticos, conducta tomada con evidencia de baja calidad, poco entendimiento de la biología tumoral y ausencia de tratamientos sistémicos efectivos (6). El desarrollo de la biopsia de ganglio centinela $-y$ su introducción en el tratamiento del melanoma-, permitió una evaluación con menor morbilidad y mayor exactitud de las cadenas ganglionares regionales $(7,8)$. Con la evolución del conocimiento del comportamiento del melanoma, se evidencia controversia histórica sobre el papel de la linfadenectomía completa en el manejo de los pacientes con melanoma. Resultados de estudios clínicos aleatorizados no encontraron beneficio en la supervivencia global específica por melanoma al realizar linfadenectomía, después de la biopsia de ganglio centinela, con reporte de patología positivo $(9,10,11)$. A eso se suma que las complicaciones tardías de los vaciamientos ganglionares dejan una morbilidad importante en los pacientes, que impactan de manera directa su calidad de vida $(7,8)$ y generan retraso en el inicio del tratamiento sistémico adyuvante como consecuencia del tiempo para la realización de otro procedimiento quirúrgico, el manejo post operatorio, y en algunos casos por las complicaciones derivadas del mismo, más aun cuando las nuevas terapias (anti PD-L1 y anti BRAF) muestran beneficio en supervivencia libre de enfermedad $(12,13,14)$.

\section{Objetivos:}

- Establecer los requisitos y consideraciones clínicas para la omisión de linfadenectomía en los pacientes con melanoma de tronco y extremidades localizado y compromiso tumoral metastásico del ganglio centinela.

- Revisar la evidencia científica actual, referente al tratamiento quirúrgico ganglionar regional, en los pacientes con diagnóstico de melanoma primario localizado y biopsia de ganglio centinela con reporte de patología positivo para compromiso tumoral.

\section{Justificación:}

- El estado del ganglio centinela se ha identificado como el factor pronóstico más fuerte de supervivencia en pacientes con diagnóstico de melanoma localizado (3).

- No hay beneficio en la supervivencia global específica por melanoma, al realizar linfadenectomía regional en los pacientes con compromiso tumoral en el ganglio centinela, por lo que existe controversia en la indicación de este procedimiento actualmente (11).

- La linfadenectomía regional completa se asocia con retraso en el inicio de tratamiento sistémico y con mayor morbilidad, por lo que es importante evaluar el valor de este procedimiento (11).

- Surgimiento de terapias sistémicas con impacto en supervivencia libre de enfermedad $(12,13,14)$.

\section{Conceptos clave:}

- Ganglio centinela: Primer ganglio o ganglios de una cadena linfatica que drena un territorio tisular determinado.

- Biopsia de ganglio centinela: Procedimiento quirúrgico que permite la identificación, extracción y analisis del ganglio centinela. 
- Linfadenectomía regional completa: Procedimiento quirúrgico en el cual los ganglios linfáticos de una cadena ganglionar específica son removidos.

\section{Metodología:}

Se realizó una búsqueda de la literatura en las dos bases de datos bibliográficas más importantes como fuentes de ensayos clínicos: MEDLINE y EMBASE, utilizando una combinación de vocabulario controlado con términos del Medical Subject Headings $(\mathrm{MeSH})$, términos Emtree, para "melanoma", "lymph nodes", "lymph node excision", "sentinel lymph node", con etiquetas de campo, operadores de proximidad y operadores boleanos. Se eligieron los ensayos clínicos aleatorizados publicados en idioma inglés hasta Noviembre 2020, que evaluaran el tratamiento quirúrgico ganglionar regional en los pacientes con diagnóstico de melanoma primario localizado y biopsia de ganglio centinela con reporte de patología positivo para compromiso tumoral. La búsqueda fue realizada por el investigador principal (IFML), realizando la tamización de los artículos por título y resumen. Posteriormente, el especialista en entrenamiento encargado (IFML), expuso los ensayos clínicos elegidos en una presentación de PowerPoint, previa supervisión por uno de los docentes del servicio (CLM). Esta presentación incluyó una revision académica de los puntos más importantes de cada ensayo clínico elegido. Se realizó una reunión inicial con la participación de las UF de Mama y Tejidos Blandos, Oncología Clínica y Patología, y una segunda reunión al interior de la UF de Mama y Tejidos Blandos, donde se socializó la evidencia científica existente, se discutieron puntos de discrepancia y se establecieron conductas a seguir en el abordaje terapéutico en relación con la omisión de linfadenectomía ganglionar en los pacientes con melanoma de tronco y extremidades con ganglio centinela positivo.

\section{Resumen de la evidencia disponible:}

En el año 2006 se publicó el estudio MSLT - I (First Multicenter Selective Lymphadenectomy Trial) (15, $16)$, y sus resultados finales en 2014. Este ensayo incluyó pacientes entre los años 1994 y 2002, con diagnóstico de melanoma cutáneo primario localizado en cabeza, cuello, tronco, extremidades o subungueal, sin compromiso ganglionar regional clínicamente evidente, Breslow mayor o igual a
$1 \mathrm{~mm}$ o Clark mayor o igual a IV, sin tratamiento quirúrgico previo. En el análisis se incluyeron 1.269 pacientes con Breslow 1.2 - 3.5mm (melanoma grosor intermedio), los cuales fueron aleatorizados a resección local amplia del tumor primario y biopsia del ganglio centinela (grupo biopsia) o resección local amplia y observación post operatoria de la cadena regional ganglionar (grupo observación). En el grupo de observación se realizaba linfadenectomía en caso de detectar, en el seguimiento, recurrencia clínicamente evidente, mientras que en el grupo de biopsia se realizaba linfadenectomía inmediata cuando el reporte del ganglio centinela era positivo para compromiso tumoral. El objetivo principal era evaluar la utilidad de la biopsia de ganglio centinela en la identificación de pacientes con metástasis ganglionares clínicamente ocultas y evaluar el efecto clínico de la linfadenectomía regional ganglionar inmediata en los pacientes con compromiso tumoral en el ganglio centinela. La supervivencia específica para melanoma y supervivencia libre de recurrencia a 10 años fue de $81.4 \%$ vs. $78.3 \%$ (HR 0.84;95\% IC 0.64$1.09 ; \mathrm{p}=0.18$ ), y de $71.3 \%$ vs. $64.7 \%$ (HR $0.76 ; 95 \%$ IC 0.62-0.94; $P=0.01$ ) para el grupo de biopsia vs. el grupo de observación respectivamente. El MSLT - I no demostró diferencias estadísticamente significativas en la supervivencia específica para melanoma en la población general del grupo biopsia vs. el grupo observación; sin embargo, evidenció mayor supervivencia libre de recurrencia estadísticamente significativa en el grupo de biopsia. En el análisis por subgrupos, los pacientes con melanoma de grosor intermedio con compromiso tumoral en el ganglio centinela vs. el grupo observación, se encontró diferencia estadísticamente significativa en la supervivencia específica para melanoma a 10 años de $62.1 \%$ vs. $41.5 \%$ (HR 0.56; IC 0.37-0.84; P= 0.006 ) respectivamente, y la supervivencia libre de enfermedad a distancia mejoró cuando los pacientes con compromiso ganglionar fueron sometidos a linfadenectomía inmediata vs. linfadenectomía tardía (HR 0.62; IC 0.42-0.91; $P=0.02$ ). En el análisis multivariado, el compromiso metastásico del ganglio centinela fue el predictor mas fuerte de recurrencia y muerte asociada a melanoma. Es de resaltar que este estudio no fue diseñado para evaluar ni comparar la utilidad de la linfadenectomía ganglionar inmediata vs. diferida posterior a identificación de compromiso tumoral en ganglio centinela; además, el análisis por subgrupos no fue planeado inicialmente y el analisis estadístico es propio del estudio, no validado en otros escenarios $(17,18)$. 
A pesar de los resultados del MSLT - I, donde quedó establecido el papel pronóstico del ganglio centinela en el enfoque de los pacientes con melanoma cutáneo primario localizado, el papel de la linfadenectomía regional inmediata, posterior a la identificación de compromiso ganglionar tumoral en el ganglio centinela, continuaba siendo un interrogante que dio paso a dos ensayos clínicos aleatorizados, multicéntricos: DeCOG - SLT (German Dermatologic Cooperative Oncology Group Selective Lymphadenectomy Trial) y MSLT - II (Second Multicenter Selective Lymphadenectomy Trial).

El ensayo clínico DeCOG - SLT $(9,10)$, incluyó pacientes entre el año 2006 al año 2014, con diagnóstico de melanoma cutáneo primario localizado de tronco y extremidades, sin compromiso ganglionar regional clínicamente evidente, Breslow mayor o igual a $1 \mathrm{~mm}$, sin tratamiento quirúrgico previo y micrometástasis en el ganglio centinela. En el análisis se incluyeron 473 pacientes, los cuales se aleatorizaron en dos grupos: el grupo de intervención sometido a linfadenectomía ganglionar regional inmediata y el grupo observación. En el grupo de observación se realizaba linfadenectomía tardía en caso de detectar recurrencia en el seguimiento. El seguimiento fue similar en los dos grupos, incluyendo examen clínico, ecografía ganglionar regional y exámenes de laboratorio cada tres meses durante los primeros tres años. El objetivo primario del estudio fue evaluar la supervivencia libre de enfermedad a distancia. En el análisis final, reportado en septiembre de 2019, con un seguimiento medio de 72 meses, se encontró que el $65.7 \%$ de los pacientes tenían compromiso ganglionar tumoral menor o igual a $1 \mathrm{~mm}$, el $19.2 \%$ entre $1 \mathrm{y}$ $2 \mathrm{~mm}$, y el $6.3 \%$ mayor a $2 \mathrm{~mm}$. La supervivencia libre de enfermedad a distancia, supervivencia global y supervivencia libre de recurrencia a cinco años en los grupos de linfadenectomía ganglionar inmediata y observación fue de $64.9 \%$ vs. $67.6 \%$ (HR 1.08, 90\% IC $0.83-1.39 ; p=0.65$ ), $72.3 \%$ vs. $71.4 \%$ (HR $0.99,90 \%$ IC $0.74-1.31 ; p=0.94$ ) y $59.9 \%$ vs. $60.9 \%$ (HR 1.01 , $90 \%$ IC $0.80-1.28, p=0.94$ ) respectivamente. Se realizó análisis por subgrupos de acuerdo a la carga tumoral del ganglio centinela, menor o igual a $1 \mathrm{~mm}$ y mayor a $1 \mathrm{~mm}$, para evaluar la supervivencia libre de enfermedad a distancia entre los dos grupos, sin diferencias estadísticamente significativas (HR $1.12,90 \%$ IC $0.80-1.57, p=0.58$ y HR $0.98,90 \%$ IC 0.63 - 1.53; $p=0.95$ ) respectivamente. En el análisis multivariado, la carga tumoral, Breslow y la ulceración fueron asociados con mayor riesgo de metástasis a distancia, supervivencia global y supervivencia libre de recurrencia.

Los resultados de este estudio confirman que la linfadenectomía ganglionar regional inmediata, en pacientes con compromiso ganglionar tumoral en el ganglio centinela, no es superior a la observación en cuanto a supervivencia libre de enfermedad a distancia, supervivencia libre de recurrencia y supervivencia global.

El otro ensayo clínico es el MSLT - II (11), que incluyó a pacientes con diagnóstico de melanoma cutáneo primario localizado en cabeza, cuello, tronco, extremidades o subungueal, sin compromiso ganglionar regional clínicamente evidente, Breslow mayor o igual a $1 \mathrm{~mm}$ o Clark mayor o igual a IV, sin tratamiento quirúrgico previo y ganglio centinela positivo para metástasis Se incluyeron 1.939 pacientes, los cuales se aleatorizaron en dos grupos: el grupo de intervención sometido a linfadenectomía ganglionar regional inmediata y el grupo de observación, en el que se realizaba linfadenectomía tardía en caso de detectar recurrencia durante el tiempo de seguimiento. Los pacientes asignados al grupo de observación tenían seguimiento clínico y ecográfico de la cadena ganglionar cada 4 meses durante los primeros 2 años, cada 6 meses hasta los 5 años y luego cada año. El estudio no encontró diferencias en la supervivencia específica para melanoma a 3 años: $86.0 \%$ para el grupo de intervención vs. $86.0 \%$ para el grupo observación (HR1.08, 95\% IC 0.88 - 1.34, $\mathrm{P}=0.42$ ). No hubo diferencias estadísticamente significativas en la supervivencia libre de metástasis a distancia entre los dos grupos (HR 1.1, 95\% IC 0.92- 1.31, $\mathrm{P}=0.31$ ). La supervivencia libre de recurrencia a 3 años fue de $68.0 \%$ en el grupo de intervención vs. $63.0 \%$ en el grupo observación ( $p=0.05)$; esta diferencia es el resultado de la reducción de recurrencia ganglionar regional en el grupo de intervención posterior a la linfadenectomía ganglionar regional inmediata de $92.0 \%$ vs. $77.0 \%$ en el grupo observación (HR 0.31, IC $0.21-0.41, p<0.001)$. Estos efectos se mantuvieron en los grupos en los cuales se identificó compromiso ganglionar tumoral mediante técnica de PCR. En el análisis multivariado, el Breslow y la ulceración fueron los factores pronósticos más importantes para muerte asociada a melanoma en los dos grupos. Se encontró compromiso metastásico de otros ganglios no centinelas en el $11.5 \%$ de los pacientes sometidos a linfadenectomía inmediata. Se presentó linfedema en $\mathbf{2 4 . 1 \%}$ de los pacientes en el grupo de 
linfadenectomía vs. $6.3 \%$ en el grupo de observación $(p<0.001)$. El estudio concluyó que la linfadenectomía inmediata en pacientes con ganglio centinela positivo no aumenta la supervivencia específica para melanoma, comparado con linfadenectomía electiva a la recaida en pacientes del grupo observación, e incrementó la tasa de control ganglionar regional pero sin impacto en la supervivencia específica para melanoma en los pacientes con compromiso tumoral en el ganglio centinela.

Dentro de las observaciones a estos ensayos clínicos, el DeCOG - SLT no alcanzó el reclutamiento ni la tasa de eventos propuestos en el diseño inicial del estudio. Sin embargo, en el análisis final para septiembre de 2019, con un mayor seguimiento, los datos son consistentes con el no beneficio del vaciamiento inmediato en pacientes con ganglio centinela positivo. El ensayo clínico MSLT - II es un estudio más robusto, en el cual el reclutamiento sí se alcanzó según el protocolo inicial, con un diseño muy similar al DeCOG - SLT. En ninguno de los dos se demostró beneficio en la supervivencia específica para melanoma con la linfadenectomía temprana vs. observación en los pacientes con ganglio centinela positivo. En el MSLT II, se encontró mayor tasa de recurrencia regional ganglionar en el grupo de observación, lo cual es un resultado esperado que también se ha evidenciado en situaciones similares en otras enfermedades, como el cáncer de mama, sin que esto tenga impacto en la supervivencia global de estos pacientes. La linfadenectomía se asocia con mayor morbilidad de la extremidad y las complicaciones derivadas del procedimiento quirúrgico pueden retrasar el inicio del tratamiento sistémico adyuvante; por lo tanto, la observación clínica, ecográfica y la linfadenectomía electiva en caso de presentar recurrencia, permitirán a los pacientes mayor tiempo libre de estas complicaciones y mejor calidad de vida. Es importante mencionar que los pacientes en el MSLT II y el DeCOG - SLT recibieron tratamiento adyuvante con interferón en un $7.2 \%$ y un $60.2 \%$ respectivamente, siendo esta terapia la única opción adyuvante para la época en la cual se realizaron los estudios. En los últimos años, se han publicado nuevos tratamientos sistémicos adyuvantes, como la inmunoterapia y la terapia dirigida, los cuales han mostrado beneficio estadísticamente significativo en supervivencia libre de enfermedad a distancia y se espera un posible beneficio en supervivencia global con un mayor seguimiento en dichos estudios $(12,13,14)$.
El estudio que permita analizar diferencias en los desenlaces clínicos de los pacientes con ganglio centinela positivo, sometidos a linfadenectomía ganglionar inmediata vs. linfadenectomía ganglionar diferida -cuando se presente recurrencia regional después de recibir tratamiento sistémico adyuvante con inmunoterapia o terapia dirigida- no ha sido diseñado y probablemente no se realice, dado que cualquier posible beneficio de la linfadenectomía inmediata sería "diluido" por el beneficio clínico en la supervivencia especifica por melanoma del tratamiento sistémico adyuvante con inmunoterapia.

Por esta razón, debemos analizar la evidencia actual para realizar consensos en pro de mejorar el manejo de los pacientes con melanoma con enfermedad ganglionar, disminuyendo el uso de tratamientos que no tienen impacto en la supervivencia específica por melanoma, los cuales pueden generar morbilidad aguda al retrasar la adyuvancia sistémica efectiva, y morbilidad crónica con disminución importante en la calidad de vida de estos pacientes.

La tabla 1 resume los aspectos mas relevantes de cada articulo.

\section{Conducta establecida en la Unidad} Funcional del INC

La decisión de omisión de linfadenectomía ganglionar regional inmediata en los pacientes con melanoma cutáneo localizado y compromiso tumoral ganglionar en la biopsia de ganglio centinela, debe ser individualizada y se deben tener en cuenta las siguientes consideraciones:

- Análisis completo de la patología del ganglio centinela, que incluya tamaño de la metástasis, número de ganglios y extensión extracapsular.

- Estandarizar el seguimiento ecográfico de las cadenas ganglionares cada 4 meses los primeros dos años, luego cada 6 meses hasta los 5 años y posteriormente cada año.

- Garantizar el seguimiento del paciente.

- Explicar al paciente la conducta terapéutica, el mayor riesgo de recaída ganglionar regional (15\% aproximadamente) sin impacto en su supervivencia, mayor morbilidad de la linfadenectomía y la importancia del seguimiento clínico y ecográfico. 
Tabla 1. Ensayos clínicos de omisión de linfadenectomía en pacientes con melanoma y ganglio centinela positivo.

\begin{tabular}{|c|c|c|}
\hline Ensayo clínico & MSLT - II (11) & DeCOG - SLT $(9,10)$ \\
\hline Diseño del estudio & Prospectivo, aleatorizado, multi-céntrico & Prospectivo, aleatorizado, multicéntrico \\
\hline Criterios de inclusión & $\begin{array}{l}\text {-Melanoma cutáneo localizado. } \\
\text {-Ganglio centinela con compromiso tumoral (H\&E, } \\
\text { IHC, PCR). } \\
\text {-Expectativa de vida > } 10 \text { años. }\end{array}$ & $\begin{array}{l}\text {-Melanoma cutáneo localizado. } \\
\text {-Breslow > } 1 \mathrm{~mm} \text {. } \\
\text {-Ganglio centinela con evidencia de } \\
\text { micrometástasis. }\end{array}$ \\
\hline Criterios de exclusión & $\begin{array}{c}\text {-Melanoma previo o recurrente. } \\
\text {-Metástasis en tránsito, regional o a distancia. } \\
\text {-Extensión extracapsular. } \\
\text {-Inmunosupresión. }\end{array}$ & $\begin{array}{l}\text {-Melanoma de cabeza y cuello. } \\
\text {-Melanoma previo, recurrente o evidencia de } \\
\text { otro cáncer. } \\
\text {-Metástasis én tránsito, regional o a distancia. } \\
\text {-Macrometástasis o extensión extracapsular. } \\
\text {-Inmunosupresión. }\end{array}$ \\
\hline Población incluida & $\begin{array}{l}\text {-1934: Intención a tratar. } \\
\text {-967: Linfadenectomia regional. } \\
\text { Reclutados en } 63 \text { centros interna-cionales* }\end{array}$ & $\begin{array}{l}\text {-473: intención a tratar. } \\
-242: \text { linfadenectomía regional. } \\
\text { Reclutados de } 41 \text { centros de cáncer de piel de } \\
\text { Alemania. }\end{array}$ \\
\hline Intervención & $\begin{array}{l}\text { Linfadenectomía inmediata vs. Observación de } \\
\text { cadena ganglionar con ecografía. }\end{array}$ & $\begin{array}{l}\text { Linfadenectomía inmediata vs. observa-ción de } \\
\text { cadena ganglionar con ecografía }\end{array}$ \\
\hline Seguimiento & 43 meses & 72 meses \\
\hline Breslow (Media) & $2.1 \mathrm{~mm}$ & $2.4 \mathrm{~mm}$ \\
\hline $\begin{array}{l}\text { Ganglio no centinela } \\
\text { positivo } \%\end{array}$ & 11.5 & 25 \\
\hline Desenlace primario & Supervivencia específica para melanoma ( $86 \%$ vs. $86 \%$ ) & $\begin{array}{l}\text { Supervivencia libre de metástasis a dis-tancia } \\
\qquad(64.9 \% \text { vs. } 67.6)\end{array}$ \\
\hline $\begin{array}{l}\text { Supervivencia libre de } \\
\text { enfermedad }\end{array}$ & $68 \%$ vs. $63 \%$. p: 0.05 a 3 años. & $59.9 \%$ vs. $60.9 \% . p=0.94$ a 5 años. \\
\hline $\begin{array}{l}\text { Control de la enfermedad } \\
\text { ganglionar }\end{array}$ & $92 \%$ vs. $77 \%$ (p: 0.001 ) control ganglionar a los 3 años. & $10.8 \%$ vs. $16.3 \%$ recurrencia ganglionar regional. \\
\hline $\begin{array}{l}\text { Morbilidad } \\
\text { 1. Linfedema } \\
\text { 2. Infección de herida }\end{array}$ & $\begin{array}{l}\text { 1. } 24.1 \text { vs. } 6.3 \% \\
\text { 2. No registrado. }\end{array}$ & $2.1 .2 \%$ \\
\hline
\end{tabular}

Modificado de Surg Clin N Am - (2019) Nonsurgical Management of Lymph Node Basins in Melanoma (19)

*Estados unidos, Australia, Alemania, Canada, España, Finlandia, Israel, Italia, Holanda, Suecia, Suiza y el Reino unido.

- Linfadenectomía terapéutica inmediata, de llegar a presentarse la recurrencia ganglionar, siempre y cuando el paciente no tenga enfermedad sistémica.

- Asegurar el inicio temprano de la terapia sistémica adyuvante.

\section{Responsabilidades éticas}

\section{Protección de personas y animales.}

Los autores declaran que, por ser este un artículo de revisión de evidencia científica, no hay pacientes involucrados.

\section{Derecho a la privacidad y consentimiento informado.}

No ha sido necesario obtener consentimientos informados.

\section{Fuente de financiación}

Ninguna.

\section{Conflicto de intereses}

Ninguno de los autores tiene conflictos de interés. 


\section{Referencias}

1. [Internet] Gco.iarc.fr. 2021. [cited 10 January 2021]. Available from: <https://gco.iarc.fr/today/data/factsheets/cancers/16Melanoma-of-skin-fact-sheet.pdf>

2. Pozzobon F, Acosta Á, Castillo J. Cáncer de piel en Colombia: cifras del Instituto Nacional de Cancerología. Revista de la Asociación Colombiana de Dermatología y Cirugía Dermatológica. 2018;26(1):12-17.

3. Angeles C, Wong S. Management of Regional Nodal Melanoma. Surgical Oncology Clinics of North America. 2020;29(3):415431. https://doi.org/10.1016/j.soc.2020.02.007

4. Landry C, McMasters $\mathrm{K}$, Scoggins $\mathrm{C}$. The evolution of the management of regional lymph nodes in melanoma. Journal of Surgical Oncology. 2007;96(4):316-321. https://doi.org/ $10.1002 /$ jso. 20867

5. Cascinelli N, Morabito A, Santinami M, MacKie R, Belli F. Immediate or delayed dissection of regional nodes in patients with melanoma of the trunk: a randomised trial. The Lancet. 1998;351(9105):793-796. https://doi.org/10.1016/s01406736(97)08260-3

6. Balch C, Soong S, Bartolucci A, Urist M, Karakousis C, Smith T et al. Efficacy of an Elective Regional Lymph Node Dissection of 1 to $4 \mathrm{~mm}$ Thick Melanomas for Patients 60 Years of Age and Younger. Annals of Surgery. 1996;224(3):255-266. https://doi. org/10.1097/00000658-199609000-00002

7. Thompson J, McCarthy W, Bosch C, O??Brien C, Quinn M, Paramaesvaran $S$ et al. Sentinel lymph node status as an indicator of the presence of metastatic melanoma in regional lymph nodes. Melanoma Research. 1995;5(4):255-260. https:// doi.org/10.1097/00008390-199508000-00008

8. Morton D, Thompson J, Essner R, Elashoff R, Stern S, Nieweg $O$ et al. Validation of the Accuracy of Intraoperative Lymphatic Mapping and Sentinel Lymphadenectomy for Early-Stage Melanoma. Annals of Surgery. 1999;230(4):453. https://doi. org/10.1097/00000658-199910000-00001

9. Leiter U, Stadler R, Mauch C, Hohenberger W, Brockmeyer N, Berking $C$ et al. Complete lymph node dissection versus no dissection in patients with sentinel lymph node biopsy positive melanoma (DeCOG-SLT): a multicentre, randomised, phase 3 trial. The Lancet Oncology. 2016;17(6):757-767. https://doi. org/10.1016/S1470-2045(16)00141-8

10. Leiter U, Stadler R, Mauch C, Hohenberger W, Brockmeyer N, Berking $C$ et al. Final Analysis of DeCOG-SLT Trial: No Survival Benefit for Complete Lymph Node Dissection in Patients With Melanoma With Positive Sentinel Node. Journal of Clinical Oncology. 2019;37(32):3000-3008. https://doi.org/10.1200/ JCO.18.02306

11. Faries M, Thompson J, Cochran A, Andtbacka R, Mozzillo $\mathrm{N}$, Zager J et al. Completion Dissection or Observation for Sentinel-Node Metastasis in Melanoma. New England Journal of Medicine. 2017;376(23):2211-2222. https://doi.org/10.1056/ NEJMoa1613210
12. Long $G$, Hauschild A, Santinami $M$, Atkinson V, Mandalà $M$, Chiarion-Sileni $\vee$ et al. Adjuvant Dabrafenib plus Trametinib in Stage IIIBRAF-Mutated Melanoma. New England Journal of Medicine. 2017;377(19):1813-1823. https://doi.org/10.1056/ NEJMoa1708539

13. Weber J, Mandala M, Del Vecchio M, Gogas H, Arance A, Cowey $C$ et al. Adjuvant Nivolumab versus Ipilimumab in Resected Stage III or IV Melanoma. New England Journal of Medicine. 2017;377(19):1824-1835. https://doi.org/10.1056/ NEJMoa1709030

14. Eggermont A, Blank C, Mandala M, Long G, Atkinson V, Dalle $S$ et al. Adjuvant Pembrolizumab versus Placebo in Resected Stage III Melanoma. New England Journal of Medicine. 2018;378(19):1789-1801. https://doi.org/10.1056/ NEJMoa1802357

15. Morton D, Thompson J, Cochran A, Mozzillo N, Elashoff R, Essner R et al. Sentinel-Node Biopsy or Nodal Observation in Melanoma. New England Journal of Medicine. 2006;355(13):1307-1317. https://doi.org/10.1056/NEJMoa060992

16. Morton D, Thompson J, Cochran A, Mozzillo N, Nieweg O, Roses $D$ et al. Final Trial Report of Sentinel-Node Biopsy versus Nodal Observation in Melanoma. New England Journal of Medicine. 2014;370(7):599-609. https://doi.org/10.1056/NEJMoa1310460

17. Thomas J. Concerns relating to the conduct and statistical analysis of the Multicenter Selective Lymphadenectomy Trial (MSLT-1) in patients with melanoma. Journal of Plastic, Reconstructive \& Aesthetic Surgery. 2009;62(4):442-446. https://doi.org/ 10.1016/j.bjps.2009.01.027

18. Altstein L, Li G. Latent Subgroup Analysis of a Randomized Clinical Trial through a Semiparametric Accelerated Failure Time Mixture Model. Biometrics. 2013;69(1):52-61. https:// doi. org/10.1111/j.1541-0420.2012.01818.x

19. Habashi R, Francescutti V. Nonsurgical Management of Lymph Node Basins in Melanoma. Surgical Clinics of North America. 2020;100(1):91-107. https://doi.org/ 10.1016/j. suc.2019.09.008 